\title{
Prospective, randomized comparison of gadopentetate and gadobutrol to assess chronic myocardial infarction applying cardiovascular magnetic resonance
}

\author{
Andre Rudolph ${ }^{1,2^{*}}$, Daniel Messroghli ${ }^{3}$, Florian von Knobelsdorff-Brenkenhoff ${ }^{1,2}$, Julius Traber ${ }^{1,2}$, \\ Johannes Schüler ${ }^{1,2}$, Ralf Wassmuth ${ }^{1,2}$ and Jeanette Schulz-Menger ${ }^{1,2}$
}

\begin{abstract}
Background: We hypothesized that the contrast medium gadobutrol is not inferior compared to Gd-DTPA in identifying and quantifying ischemic late gadolinium enhancement (LGE), even by using a lower dose.

Methods: We prospectively enrolled 30 patients with chronic myocardial infarction as visualized by LGE during clinical routine scan at $1.5 \mathrm{~T}$ with $0.20 \mathrm{mmol} / \mathrm{kg}$ Gd-DTPA. Participants were randomized to either $0.15 \mathrm{mmol} / \mathrm{kg}$ gadobutrol (group A) or $0.10 \mathrm{mmol} / \mathrm{kg}$ gadobutrol (group B). CMR protocol was identical in both exams. LGE was quantified using a semiautomatic approach. Signal intensities of scar, remote myocardium, blood and air were measured. Signal to noise (SNR) and contrast to noise ratios (CNR) were calculated.

Results: Signal intensities were not different between Gd-DTPA and gadobutrol in group A, whereas significant differences were detected in group B. SNR of injured myocardium (53.5+/-21.4 vs. 30.1+/-10.4, $p=0.0001$ ) and CNR between injured and remote myocardium $(50.3+/-20.3$ vs. $27.3+/-9.3, p<0.0001)$ were lower in gadobutrol. Infarct size was lower in both gadobutrol groups compared to Gd-DTPA (group A: 16.8+/-10.2 g vs. 12.8+/-6.8 g, $p=0.03$; group B: $18.6+/-12.0 \mathrm{~g}$ vs. $14.0+/-9.9 \mathrm{~g}, p=0.0016)$.
\end{abstract}

Conclusions: Taking application of $0.2 \mathrm{mmol} / \mathrm{kg}$ Gd-DTPA as the reference, the delineation of infarct scar was similar with $0.15 \mathrm{mmol} / \mathrm{kg}$ gadobutrol, whereas the use $0.10 \mathrm{mmol} / \mathrm{kg}$ gadobutrol led to reduced tissue contrast.

Trial registration: The study had been registered under EudraCT Number: 2010-020775-22. Registration date: 2010.08.10

Keywords: Cardiovascular Magnetic Resonance, Contrast media, Gadobutrol, Gd-DTPA, Chronic myocardial infarction, Late gadolinium enhancement

\section{Background}

Myocardial infarction is a leading cause of mortality worldwide. Accurate assessment of infarct size and morphology is important for clinical decision making in a lot of clinical settings $[1,2]$. Currently, late gadolinium enhancement cardiac magnetic resonance (LGE-CMR) is

\footnotetext{
* Correspondence: a.rudolph@charite.de

'Working Group CMR, Experimental and Clinical Research Center, a joint cooperation between the Charité Medical Faculty and the Max-Delbrück Center for Molecular Medicine, Lindenberger Weg 80, 13125 Berlin, Germany ${ }^{2}$ Dept. of Cardiology and Nephrology, HELIOS-Kliniken Berlin Buch, Schwanebecker Chaussee 50, 13125 Berlin, Germany

Full list of author information is available at the end of the article
}

a well-established and accurate method to assess scar morphology [3] and is widely used in clinical CMR $[4,5]$. Until now, Gd-DTPA, a linear ionic gadolinium chelate, is the extracellular contrast agent that is mostly used for detection of myocardial LGE and is commonly administered as double dose $(0.2 \mathrm{mmol} / \mathrm{kg})$. After the administration of Gd-DTPA, cases of nephrogenic systemic fibrosis (NSF) in patients with renal failure have been reported, leading to a new risk classification of contrast media [6]. Whereas the detailed mechanism of NSF is not known, it could be shown in animal experiments that the tissue gadolinium distribution is altered in case of renal impairment [7]. 
Gadobutrol is a macrocyclic nonionic gadolinium chelate with a higher $\mathrm{T}_{1}$ relaxivity compared to GdDTPA (gadobutrol: $\mathrm{r} 1=4.7 \mathrm{mmol}^{-1} \mathrm{~s}^{-1}$; Gd-DTPA: $\mathrm{r} 1=$ $3.9 \mathrm{mmol}^{-1} \mathrm{~s}^{-1}$, at $1.5 \mathrm{Tesla}$, in human blood plasma, at $37{ }^{\circ} \mathrm{C}$ ) [8]. The binding profile is known to be more stable, thus through to have a lower risk of NSF. Only three controversially discussed cases of NSF were reported after the use of gadobutrol worldwide until now $[9,10]$. Therefore gadobutrol is classified as a low risk contrast-medium. This and the higher relaxivity are driving forces for further evaluations in cardiovascular settings.

Today, there are only limited data available analyzing the influence of contrast agents with different relaxivities on LGE quantification. In this study, we hypothesized that gadobutrol, which is characterized by a higher relaxivity compared to Gd-DTPA, is not inferior to GdDTPA in identifying and quantifying ischemic LGE, even when using a lower dose.

\section{Methods}

\section{Patients' enrollment}

Between September 2010 and December 2011, patients with a history of a chronic myocardial infarction and LGE as assessed during a clinical CMR scan using $0.20 \mathrm{mmol} / \mathrm{kg}$ Gd-DTPA were screened and asked for participation in a second study-related CMR scan. All included patients gave written informed consent and were scheduled for a second CMR examination afterwards. The study had been registered under EudraCT number: 2010-020775-22 and was accordingly approved by the ethical board of the city of Berlin (LAGESO).

The sample size of this randomized prospective trial was calculated based on a power calculation. The assumption was, that for a two-sided alpha of $5 \%$ and a power of $80 \%$ based on a delta of $0.15,15$ patients per dose gadobutrol have to be included.

Patients were randomized for either $0.15 \mathrm{mmol} / \mathrm{kg}$ (group A) or $0.10 \mathrm{mmol} / \mathrm{kg}$ (group B) gadobutrol. All patients were in normofrequent sinus rhythm.

\section{Inclusion criteria}

- between 18 and 80 years of age

- chronic myocardial infarction (>3 months old) based on coronary artery disease (CAD), detected by invasive angiography

- successfully performed clinical LGE CMR study within the last 4 weeks

- evidence of single myocardial infarction on LGE CMR with "hyper-enhancement" involving at least two contiguous short-axis slices

- written informed consent

\section{Exclusion criteria}

- severe arrhythmia, atrial fibrillation

- history of moderate or severe impairment of renal function (GFR $<60 \mathrm{ml} / \mathrm{min})$

- additional myocardial infarction or acute coronary syndrome during the last 4 weeks

- general contraindications for CMR

\section{Image acquisition}

CMR was performed on a 1.5 Tesla cardiac-dedicated clinical MR system (Avanto, Siemens Healthcare, Erlangen, Germany) using a 12-channel coil. Identical CMR protocols were applied for both examinations.

LGE LGE was assessed in all patients starting $13 \mathrm{~min}$ after contrast administration in a short axis stack by using a $2 \mathrm{D}$ inversion recovery gradient echo sequence (slice thickness $8 \mathrm{~mm}$, no gap, TE $5.0 \mathrm{~ms}$, FA $30^{\circ}$, matrix $256 \times 192$, field of view $350 \times 262 \mathrm{~mm}$ ) with an inversion time (TI) adjusted to null signal from normal myocardium. In case of questionable bright signal in the myocardium during the scan, we changed the read out or acquired a perpendicular slice to exclude artefacts. Slice position was identical as for cine imaging using a single slice single breathold approach.

$T_{1}$ quantification $T_{1}$ quantification was performed with a Modified Look-Locker Inversion-recovery (MOLLI) sequence [11], acquired in a mid-ventricular short-axis slice before and 1, 3, 5, 9, 20 min after contrast administration. Multiple post-contrast measurements were performed to show the optimal time point of image acquisition after contrast administration. Imaging parameters were: nonselective inversion pulse, steady-state free precession single-shot read out in mid-diastole, field of view $223 \times$ $320 \mathrm{~mm}$, matrix $174 \times 192$, slice thickness $8 \mathrm{~mm}$, TE $1.08 \mathrm{~ms}$, FA $35^{\circ}$, bandwidth $1002 \mathrm{~Hz} / \mathrm{Px}$, minimum inversion time of $100 \mathrm{~ms}$, maximum inversion time of $3600 \mathrm{~ms}$.

\section{Image analysis}

Function For analyzing LV function and volumes, the endocardial and epicardial contours were manually drawn in systole and diastole using dedicated software $\left(\mathrm{CMR}^{42}\right.$, circle, Calgary, Canada). LV mass was calculated as total myocardial volume multiplied by the specific gravity of the myocardium $(1.05 \mathrm{~g} / \mathrm{ml})$. LV mass and LV end diastolic volume were indexed to height.

LGE The readers were blinded to the group assignment. For quantification of LGE a semiautomatic gray-scale threshold technique was performed as published previously 


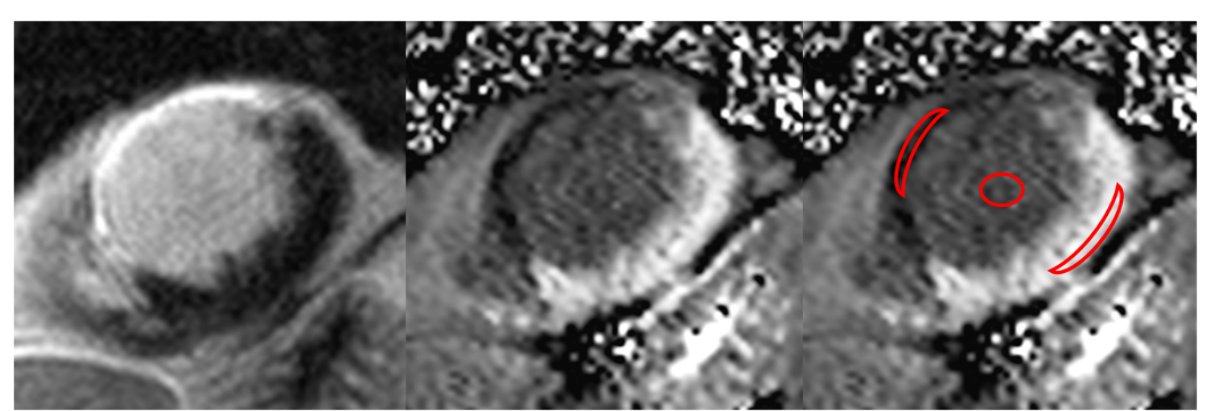

Fig. $1 T_{1}$-Mapping. LGE-image in short axis view (left) with corresponding $T_{1}$-map (center and right) show a transmural anteroseptal myocardial infarction. ROls (infarct region, remote myocardium and blood pool) were drawn on the map (right)

[12]. Areas of LGE were defined as a signal intensity of more than 6 standard deviations (SD) above the mean of remote myocardium.

Signal intensities of scar tissue, remote myocardium, blood and air were measured by drawing free-hand ROIs of approximately 10-20 pixels in each LGE short axis stack. Reproducible locations were achieved by using anatomical landmarks in dedicated software $\left(\mathrm{CMR}^{42}\right.$, circle international, Calgary Canada). For signal-to-noise ratio (SNR) and contrast-to-noise ratio (CNR) the following sequations were used [13]:

\section{$\mathrm{SNR}=$ mean signal of $\mathrm{ROI} /$ standard deviation of background noise. CNR (between tissue A} and tissue $\mathrm{B})=\operatorname{SNR}(\mathrm{A})-\mathrm{SNR}(\mathrm{B})$.

$T_{1}$ quantification $T_{1}$ maps were constructed offline using MRmap $[14,15]$ a customized software program written in Interactive Data Language (IDL; RSI International, Boulder, CO, USA). The position of the source images was initially manually adjusted to correct for potential misregistration. A curve fit of the MOLLI source images was then performed, with automatic calculation of $T_{1}$ values for each pixel. A $T_{1}$ parametric map was subsequently generated and used for further analysis. The parametric maps were evaluated in $\mathrm{CMR}^{42}$. Freehand ROI's of approximately 10-20 pixels were placed in scar tissue, remote myocardium and blood for measuring the averaged $\mathrm{T}_{1}$ time in this tissue. Reference for scar

Table 1 Patient's characteristics

\begin{tabular}{lll}
\hline & Group A & Group B \\
\hline Male/female & $15 / 0$ & $15 / 0$ \\
Age (years) & $70.20 \pm 4.70$ & $63.50 \pm 10.70$ \\
Scar amount (g) & $16.77 \pm 10.18$ & $18.63 \pm 11.98$ \\
SNR scar & $45.51 \pm 22.56$ & $53.45 \pm 21.40$ \\
CNR between scar tissue & $41.76 \pm 21.24$ & $50.27 \pm 20.30$ \\
and remote myocardium & & \\
\hline
\end{tabular}

delineation was the LGE image in the same plane (Fig. 1).

\section{Interobserver variability}

To test inter-observer variability in LGE quantification, two readers who were blinded to each other's results analyzed all examinations.

\section{Statistical analysis}

Statistical analyses were performed using SAS 9.2 (SAS Institute Inc., Cary, NC, USA) and SPSS 21.0 for windows (Chicago, Il, USA). Data are presented as mean \pm standard deviation. Statistical tests were performed using non-parametric methods due to the low sample size within each group. Continuous data were compared using the Wilcoxon rank sum tests. Paired data were compared using Wilcoxon signed rank tests. Categorical data were compared using Fisher's exact test. Statistical tests were considered significant with the two-sided $\mathrm{p}<$ 0.05. Spearman correlation coefficient's were used to determine observer-related variability. Boxplots were generated with SPSS and show median (line in the middle), 1st quartile (bottom of box), 3rd quartile (top of box), lowest case within 1.5 times IQR (bottom whisker), highest case within 1.5 times IQR (top whisker) and outliers.

Table 2 Signal intensities and LGE quantification in group A

\begin{tabular}{lccc}
\hline & $\begin{array}{l}\text { Gd-DTPA } \\
(0.2 \mathrm{mmol} / \mathrm{kg})\end{array}$ & $\begin{array}{l}\text { Gadobutrol } \\
(0.15 \mathrm{mmol} / \mathrm{kg})\end{array}$ & p-value \\
\hline SNR of scar tissue & $45.51 \pm 22.56$ & $47.04 \pm 19.78$ & $>0.9999$ \\
CNR between scar tissue & $41.76 \pm 21.24$ & $42.83 \pm 17.31$ & $>0.9999$ \\
$\begin{array}{l}\text { and remote myocardium } \\
\text { SNR of blood }\end{array}$ & $43.97 \pm 16.32$ & $44.37 \pm 16.20$ & 0.6698 \\
$\begin{array}{l}\text { CNR between scar tissue } \\
\text { and blood }\end{array}$ & $1.53 \pm 17.18$ & $2.65 \pm 14.02$ & 0.5153 \\
Amount of LGE (g) & $16.77 \pm 10.18$ & $12.84 \pm 6.79$ & 0.0300 \\
\hline
\end{tabular}




\section{Results}

We screened 252 patients and identified 51 who met our criteria. 30 patients agreed to participate in the trial and were prospectively enrolled in the study and randomized to either $0.15 \mathrm{mmol} / \mathrm{kg}$ gadobutrol (group A) or $0.10 \mathrm{mmol} / \mathrm{kg}$ gadobutrol (group B). 21 patients refused participation due to a lack of motivation or fear of repeated contrast administration. There were no significant differences between groups relating age, scar amount and signal intensities for control agent GdDTPA (Table 1). The time duration between both scans was at maximum 30 days. We took the history again to check for clinical events. In none of the patients a clinical event was existing.
No complications related to contrast administration were observed. Infarcts were detectable in all patients.

\section{Comparison of signal intensities and LGE amount}

In group A signal intensities showed no differences between Gd-DTPA and gadobutrol (Table 2, Figs. 2 and 3). Amount of LGE was lower in gadobutrol, the absolute difference was small, but reached statistical significance $(p=0.03)$.

In group B signal intensities showed significant differences between Gd-DTPA and gadobutrol. In particular SNR of injured myocardium and CNR between injured and remote myocardium were significantly lower in gadobutrol resulting in a significant smaller

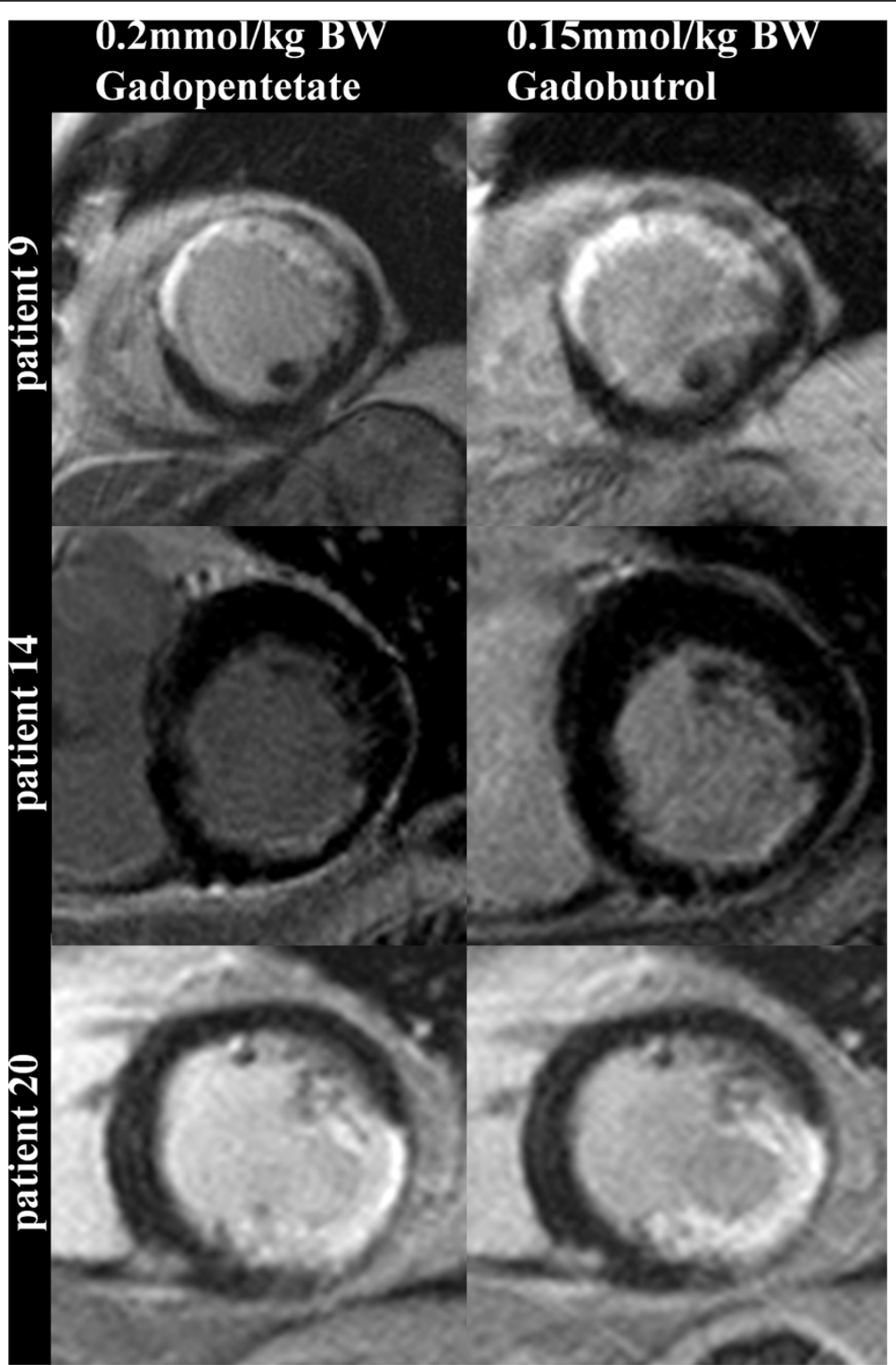

Fig. 2 LGE (short axis view) example images from group A. Left: after 0.2 mmol/kg BW Gd-DTPA. Right: after 0.15 mmol/kg BW gadobutrol 

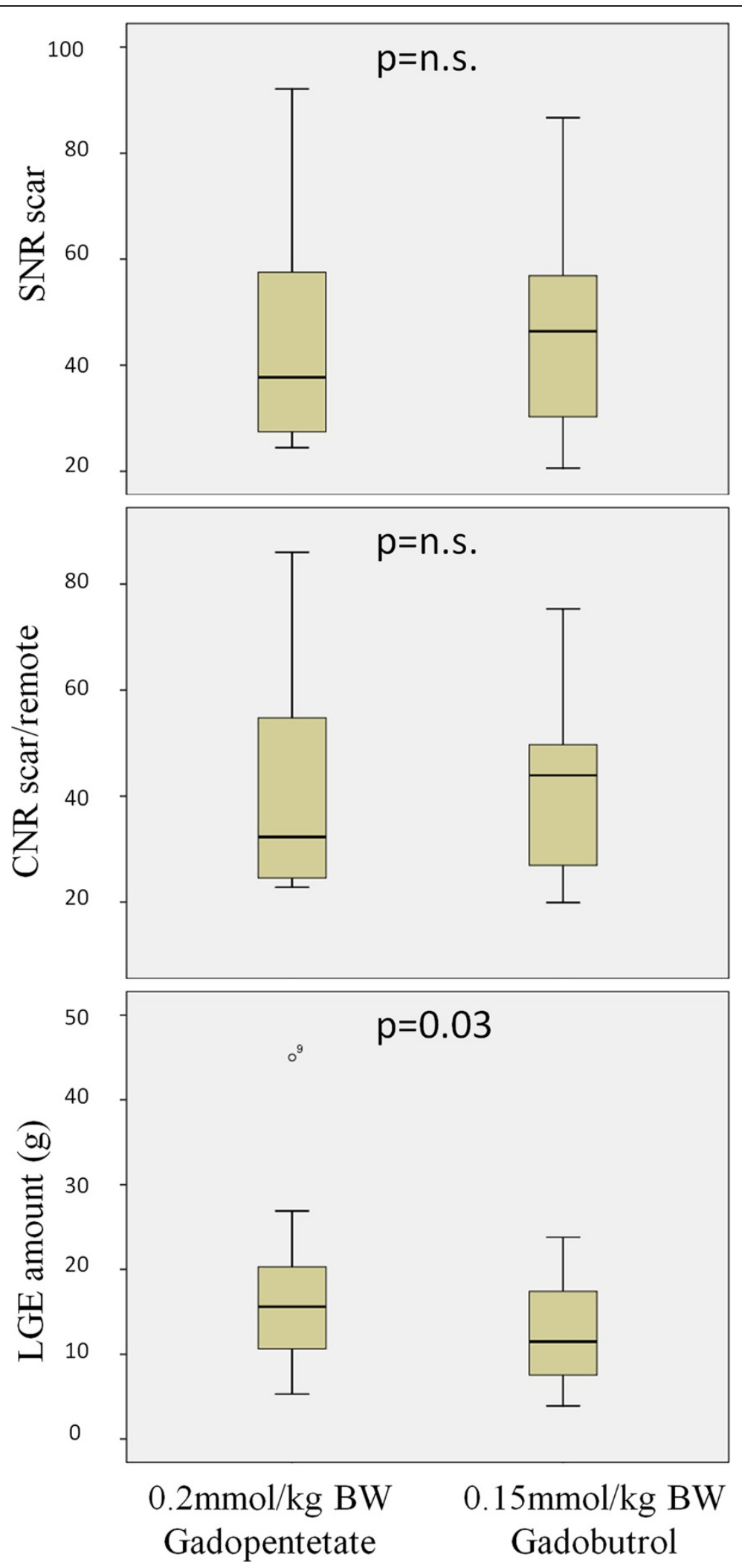

Fig. 3 Boxplots showing SNR-, CNR and LGE amount in group A. Left: after 0.2 mmol/kg BW Gd-DTPA. Right: after 0.15 mmol/kg BW gadobutrol 
Table 3 Signal intensities and LGE quantification in group B

\begin{tabular}{lccc}
\hline & $\begin{array}{l}\text { Gd-DTPA } \\
(0.2 \mathrm{mmol} / \mathrm{kg})\end{array}$ & $\begin{array}{l}\text { Gadobutrol } \\
(0.1 \mathrm{mmol} / \mathrm{kg})\end{array}$ & p-value \\
\hline SNR of scar tissue & $53.45 \pm 21.40$ & $30.11 \pm 10.36$ & 0.0001 \\
$\begin{array}{l}\text { CNR between scar tissue } \\
\text { and remote myocardium }\end{array}$ & $50.27 \pm 20.30$ & $27.32 \pm 9.28$ & $<0.0001$ \\
SNR of blood & $48.57 \pm 17.69$ & $19.84 \pm 6.33$ & $<0.0001$ \\
$\begin{array}{l}\text { CNR between scar tissue } \\
\text { and blood }\end{array}$ & $4.89 \pm 12.96$ & $10.29 \pm 9.26$ & 0.0554 \\
Amount of LGE (g) & $18.63 \pm 11.98$ & $14.03 \pm 9.92$ & 0.0016 \\
\hline
\end{tabular}

scar size as defined by the amount of LGE (Table 3, Figs. 4 and 5).

\section{Interobserver Variability of LGE quantification}

Interobserver variability was low and without differences between the contrast agents in both groups (Table 4).

\section{$T_{1}$ mapping}

For technical reasons we were able to perform a pairwise analysis in only six patients of group A. Interestingly, in

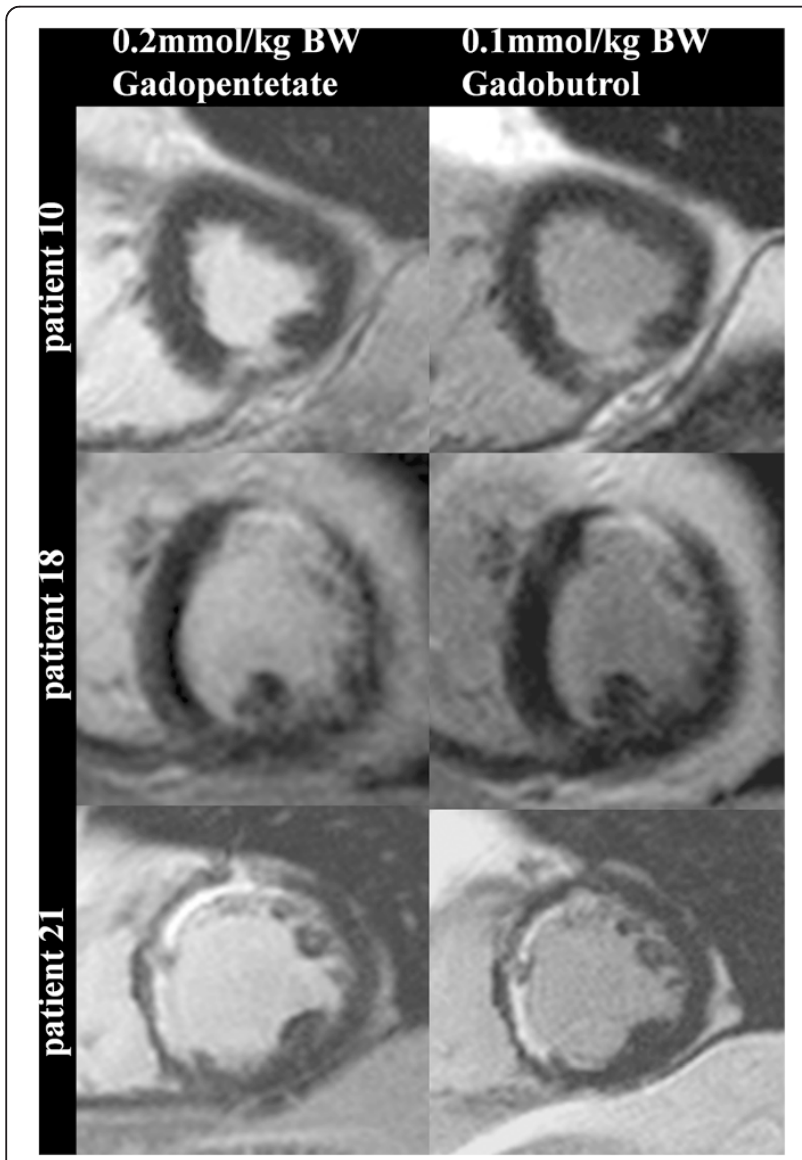

Fig. 4 LGE (short axis view) example images from group B. Left: after $0.2 \mathrm{mmol} / \mathrm{kg}$ BW Gd-DTPA. Right: after $0.1 \mathrm{mmol} / \mathrm{kg}$ BW gadobutrol this subgroup we found no significant differences between both contrast media regarding $\mathrm{T}_{1}$ times of the different tissues at different time- points after contrast administration (Table 5).

The drop-out rate was high due to two main-aspects: I) The applied sequence was a first generation one as described in the method section. II) The most frequent limitation was the registration failure of the raw images especially in regions of wall thinning respectively the infarct area.

\section{Discussion}

In the present study we compared the depiction of chronic myocardial infarction using two different contrast media and different doses in a prospective randomized setting. The null hypothesis of our study was, that the infarct volume with gadobutrol is not equivalent to the infarct volume with the control agent. This was confirmed by our results. The main results are the following:

I) Scar was detectable with each contrast media and dose. II) The use of $0.15 \mathrm{mmol} / \mathrm{kg} \mathrm{BW}$ (body weight) gadubutrol led to similar results compared to $0.2 \mathrm{mmol} /$ kg BW Gd-DTPA regarding signal intensity and contrast whereas the application of $0.1 \mathrm{mmol} / \mathrm{kg}$ gadobutrol led to a significant poorer delineation of scar tissue. III) Both doses gadobutrol led to a smaller infarct size taking $0.2 \mathrm{mmol} / \mathrm{kg}$ BW Gd-DTPA as the reference. IV) Observer variability of LGE quantification was independent from type and dose of contrast agent.

Although our sample size was small, but defined in a random setting, we could confirm in part the data by Durmus et al., who compared $0.15 \mathrm{mmol} / \mathrm{kg} \mathrm{BW}$ gadobutrol with $0.2 \mathrm{mmol} / \mathrm{kg} \mathrm{BW}$ Gd-DTPA in 20 patients with myocardial infarction [16]. They showed that gadobutrol led to similar infarct size and CNR between scar and remote myocardium compared to Gd-DTPA. In contrast to our results, CNR between scar and blood even increased with gadobutrol in their study, whereas we observed no significant difference in group A between gadubutrol and Gd-DTPA regarding SNR of blood as well as CNR between scar and blood. Our finding are supported by the quantitative parametric T1-mapping results as presented in a subgroup.

DeCobelli et al. compared gadobutrol with Gd-DTPA in patients with positive LGE regardless of its etiology [17]. They showed that $0.1 \mathrm{mmol} / \mathrm{kg} \mathrm{BW}$ gadobutrol is as effective as $0.2 \mathrm{mmol} / \mathrm{kg}$ BW Gd-DTPA regarding signal intensities and quantification of injured tissue. Our data (group B) are different to these results. In our study $0.1 \mathrm{mmol} / \mathrm{kg} \mathrm{BW}$ gadobutrol led to poorer delineation of infarct scar and reduced amount of LGE as found by both readers. A possible explanation for this discrepancy between both studies could be the different patient 

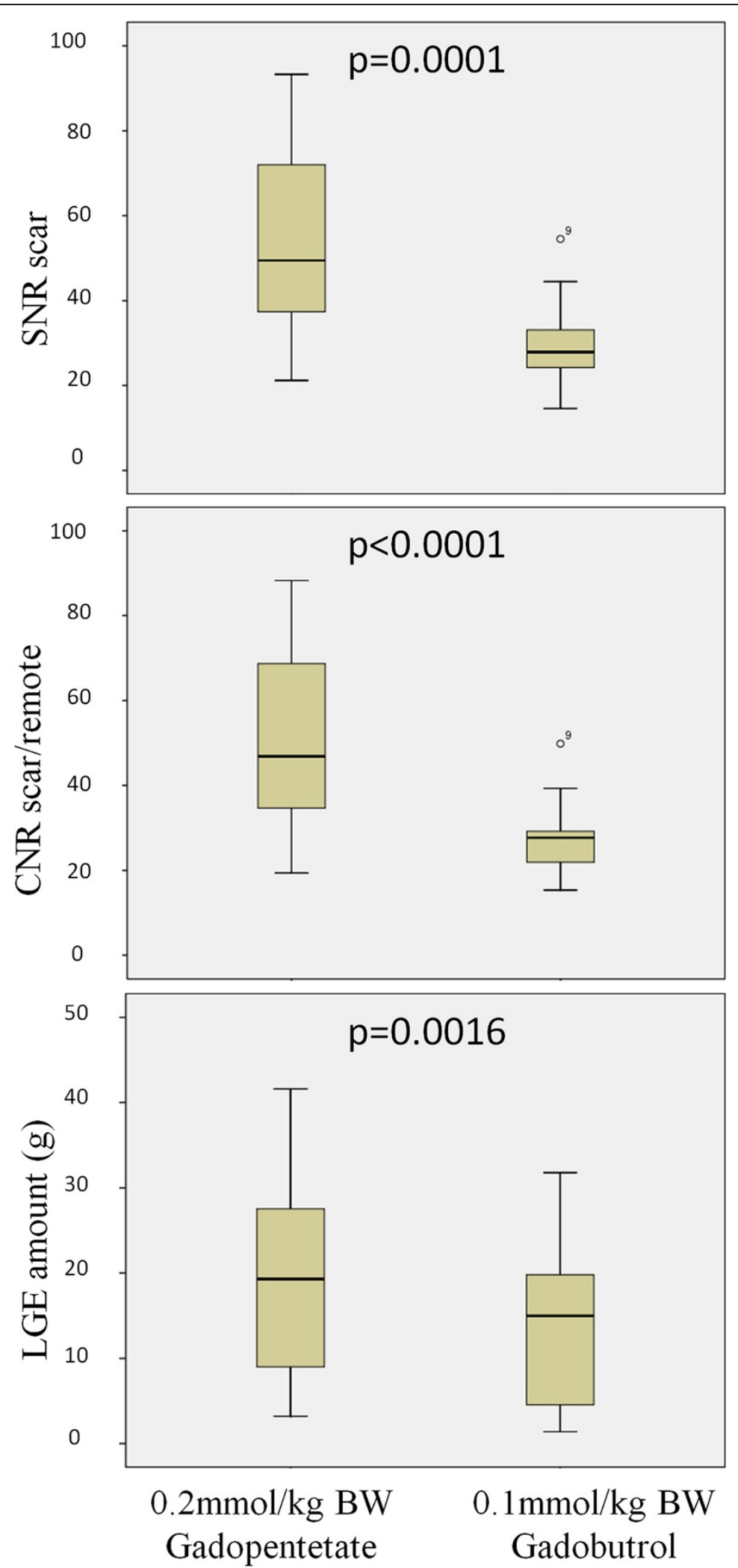

Fig. 5 Boxplots showing SNR-, CNR and LGE amount in group B. Left: after 0.2 mmol/kg BW Gd-DTPA. Right: after 0.1 mmol/kg BW gadobutrol 
Table 4 Spearman's correlation coefficients between the two readers

\begin{tabular}{lcc}
\hline & Gd-DTPA & Gadobutrol \\
\hline Group A & 0.85 & 0.93 \\
Group B & 0.91 & 0.94 \\
\hline
\end{tabular}

population. DeCobelli et al. evaluated heterogeneous groups including non-ischemic heart-diseases. It is wellknown, that the quantification in this more diffuse fibrosis is on one hand more challenging due to the small size and the blurred borders, but on the other hand the location in non-ischemic disease is typically intramural. That facilitates the differentiation from the bright blood pool signal. The different character of the fibrotic tissue in non-ischemic and ischemic heart disease seems to lead to the application of different standard-deviation for scarsizing. In case of non-ischemic heart disease, often two standard deviations were applied to differentiate LGE

Table $5 \mathrm{~T}_{1}$ values (ms) in group A $(n=6)$

\begin{tabular}{|c|c|c|c|}
\hline $\begin{array}{l}\text { Time after contrast } \\
\text { administration }\end{array}$ & $\begin{array}{l}\text { Gd-DTPA } \\
(0.2 \mathrm{mmol} / \mathrm{kgKG})\end{array}$ & $\begin{array}{l}\text { Gadobutrol } \\
(0.15 \mathrm{mmol} / \mathrm{kgKG})\end{array}$ & $\overline{p \text {-value }}$ \\
\hline \multicolumn{4}{|l|}{ Native } \\
\hline myocardium & $909 \pm 199$ & $990 \pm 117$ & 0.17 \\
\hline scar & $1033 \pm 226$ & $978 \pm 98$ & 0.92 \\
\hline blood & $1407 \pm 42$ & $1402 \pm 62$ & 0.53 \\
\hline \multicolumn{4}{|l|}{$1 \mathrm{~min}$} \\
\hline myocardium & $248 \pm 38$ & $250 \pm 36$ & 0.92 \\
\hline scar & $207 \pm 28$ & $210 \pm 36$ & 0.75 \\
\hline blood & $134 \pm 18$ & $144 \pm 15$ & 0.35 \\
\hline \multicolumn{4}{|l|}{$3 \mathrm{~min}$} \\
\hline myocardium & $305 \pm 50$ & $311 \pm 23$ & 0.75 \\
\hline scar & $227 \pm 18$ & $241 \pm 36$ & 0.35 \\
\hline blood & $181 \pm 23$ & $200 \pm 17$ & 0.25 \\
\hline \multicolumn{4}{|l|}{$5 \mathrm{~min}$} \\
\hline myocardium & $361 \pm 57$ & $360 \pm 18$ & 0.92 \\
\hline scar & $275 \pm 31$ & $285 \pm 48$ & 0.46 \\
\hline blood & $215 \pm 21$ & $248 \pm 52$ & 0.08 \\
\hline \multicolumn{4}{|l|}{$9 \min$} \\
\hline myocardium & $416 \pm 61$ & $405 \pm 22$ & 0.6 \\
\hline scar & $300 \pm 50$ & $308 \pm 47$ & 0.35 \\
\hline blood & $260 \pm 28$ & $265 \pm 21$ & 0.6 \\
\hline \multicolumn{4}{|l|}{$20 \mathrm{~min}$} \\
\hline myocardium & $472 \pm 63$ & $468 \pm 35$ & 0.89 \\
\hline scar & $351 \pm 67$ & $361 \pm 64$ & 0.5 \\
\hline blood & $330 \pm 42$ & $337 \pm 26$ & 0.5 \\
\hline
\end{tabular}

from remote myocardium [18, 19]. For clinical decision making usually visual assessment of LGE images is recommended, whereas quantitative analysis of LGE extent and/or "grey-zone" extent is common for research purposes [20]. The integration of pharmacokinetic models with different compartments could also help to reduce the influence of different contrast dynamics in different types of fibrosis [21], as well as different analysis tools [22].

One would assume that parametric mapping techniques will help to overcome that problem. Whereas mapping techniques are already applied in different diseases and also for the quantification of extracellular volumes [23, 24], no standardized approach is given today. Furthermore automatic assessment of infarct borders will help to overcome subjective approaches [25], but they also depend on the predefined gold standards.

Independent of the quantification method itself, contrast media with higher relaxivity are warranted for potential dose reduction. The concept of dose reduction in contrast agents with higher relaxivity was also analyzed for gadobenate dimeglumine. Recent studies have shown a similar diagnostic performance and delineation of infarct scars using reduced doses of gadobenate dimeglumine compared to standard dose Gd-DTPA $(0.2 \mathrm{mmol} /$ $\mathrm{kg} \mathrm{BW)} \mathrm{[26-28].} \mathrm{When} \mathrm{comparing} \mathrm{equivalent} \mathrm{doses} \mathrm{of}$ gadobenate dimeglumine with Gd-DTPA [29], higher SNR- and CNR between scar and remote myocardium was observed for gadobenate dimeglumine, whereas the CNR between scar and blood decreased resulting in poorer delineation of small subendocardial infarcts. New technical developments could help to overcome this limit. Recently, a new multi contrast LGE sequence (MCODE) was proposed to improve detection of subendocardial myocardial infarction [30].

Reduced dose of gadobutrol allows a reproducible detection of myocardial infarction in all patients. In clinical routine visual assessment of scar is accepted and eye balling is accepted [12]. But scar quantification may play an important role in treatment planning in future and is expected to play a crucial role in risk stratification $[1,31,32]$. Therefore, a simple and robust post-processing method is required. The present study demonstrated that the inter-observer variability of LGE quantification by using the 6-SD threshold yielded satisfactory results in ischemic lesions independent from the contrast type and dose. However, we observed that relaxivity and dose influence the absolute results, which underlines that accurate follow-up evaluations need constant CMR conditions in clinical trials. CMR offers the unique capability to bring reliable (semi)quantitative approaches of myocardial tissue differentiation based on contrast- and non-contrast enhanced techniques into clinical routine therefore standardization is of importance. 


\section{Limitations}

The present study was conducted at a single center with a relatively small sample size. The study only included men to omit the influence of sex on the results. Further studies in women are necessary to extend the conclusions to both sexes.

SNR- and CNR of LGE images are influenced by inversion time. Its adjustment is operator-dependent. T1mapping was expected to add information on contrastmedia timing, but for technical reasons we were only able to complete a pairwise T1 analysis in six patients of group A. Larger studies preferably with mapping techniques are needed to confirm our results.

\section{Conclusions}

The use of $0.15 \mathrm{mmol} / \mathrm{kg} \mathrm{BW}$ gadobutrol led to comparable results as $0.20 \mathrm{mmol} / \mathrm{kg}$ BW Gd-DTPA in delineation of myocardial infarction and only to a small, clinically non-relevant deviation in quantification of infarct scar and may be an appropriate alternative under consideration of the lower risk for NSF. In contrast, the dose of $0.10 \mathrm{mmol} / \mathrm{kg}$ BW gadobutrol was associated with lower signal intensity and higher discrepancy regarding infarct size. Our results underline that accurate scar evaluation during follow-up depends on type of contrast agent and contrast dose and therefore requires constant study conditions.

\section{Competing interests}

The trial was supported by a grant of Bayer HealthCare Medical Care (Bayer Pharma AG, Berlin, Germany) (See: Acknowledgements).

\section{Author' contributions}

AR participated in the design of the study, coordinated the scans, evaluated the data and drafted the manuscript, DM conceived of the study, participated in its design and helped to draft parts of the manuscript; FvK in the design of the study, evaluated part of the data, helped to draft the manuscript, JT participated in data postprocessing, partly statistical analysis. JS performed dataanalysis. RW participated in the design of the study and performed CMR scans. JSM conceived main parts of the study, participated in the design and helped to draft the manuscript. All authors read and approved the final manuscript."

\section{Acknowledgements}

The authors would like to thank our technicians, led by Kerstin Kretschel, for their assistance and great help in acquiring the images.

Furthermore, without the work of our study nurse Annette Köhler this study would not have been possible.

We are thankful for the tremendous help of Edyta Blaszczyk during the revision process.

The trial was supported by a grant of Bayer HealthCare (Bayer Pharma AG, Berlin, Germany). The authors want to thank the HELIOS research center especially Dr. Heinrich and Mr. Welcker for their help in formal work during run the applications. Prof. Petra Thürmann in handling the formal challenges.

\section{Author details}

${ }^{1}$ Working Group CMR, Experimental and Clinical Research Center, a joint cooperation between the Charité Medical Faculty and the Max-Delbrück Center for Molecular Medicine, Lindenberger Weg 80, 13125 Berlin, Germany. ${ }^{2}$ Dept. of Cardiology and Nephrology, HELIOS-Kliniken Berlin Buch, Schwanebecker Chaussee 50, 13125 Berlin, Germany. ${ }^{3}$ Department of Congenital Heart Disease and Pediatric Cardiology, Deutsches Herzzentrum Berlin, Berlin, Germany.
Received: 26 May 2015 Accepted: 9 November 2015

Published online: 17 November 2015

\section{References}

1. Boye P, Abdel-Aty H, Zacharzowsky U, Bohl S, Schwenke C, van der Geest $\mathrm{RJ}$, et al. Prediction of life-threatening arrhythmic events in patients with chronic myocardial infarction by contrast-enhanced CMR. JACC Cardiovasc Imaging. 2011;4(8):871-9.

2. de Haan S, Meijers TA, Knaapen P, Beek AM, van Rossum AC, Allaart CP. Scar size and characteristics assessed by CMR predict ventricular arrhythmias in ischaemic cardiomyopathy: comparison of previously validated models. Heart. 2011;97(23):1951-6.

3. Kim RJ, Chen EL, Lima JA, Judd RM. Myocardial Gd-DTPA kinetics determine MRI contrast enhancement and reflect the extent and severity of myocardial injury after acute reperfused infarction. Circulation. 1996;94(12):3318-26.

4. von Knobelsdorff-Brenkenhoff F, Schulz-Menger J. Cardiovascular magnetic resonance imaging in ischemic heart disease. J Magn Reson Imaging. 2012; 36(1):20-38

5. von Knobelsdorff-Brenkenhoff F, Bublak A, El-Mahmoud S, Wassmuth R, Opitz C, Schulz-Menger J. Single-centre survey of the application of cardiovascular magnetic resonance in clinical routine. Eur Heart J Cardiovasc Imaging. 2013;14(1):62-8.

6. Yang L, Krefting I, Gorovets A, Marzella L, Kaiser J, Boucher R, et al. Nephrogenic systemic fibrosis and class labeling of gadolinium-based contrast agents by the Food and Drug Administration. Radiology. 2012; 265(1):248-53.

7. Wadas TJ, Sherman CD, Miner JH, Duncan JR, Anderson CJ. The biodistribution of [153Gd] Gd-labeled magnetic resonance contrast agents in a transgenic mouse model of renal failure differs greatly from control mice. Magn Reson Med. 2010;64(5):1274-80.

8. Pintaske J, Martirosian P, Graf H, Erb G, Lodemann KP, Claussen CD, et al. Relaxivity of Gadopentetate Dimeglumine (Magnevist), Gadobutrol (Gadovist), and Gadobenate Dimeglumine (MultiHance) in human blood plasma at 0.2, 1.5, and 3 Tesla. Invest Radiol. 2006;41(3):213-21.

9. Wollanka H, Weidenmaier W, Giersig C. NSF after Gadovist exposure: a case report and hypothesis of NSF development. Nephrol Dial Transplant. 2009; 24(12):3882-4.

10. Elmholdt TR, Bettina J, Mette R, Pedersen M, Olesen AB. Two cases of nephrogenic systemic fibrosis after exposure to the macrocyclic compound gadobutrol. NDT Plus. 2010;3:285-7.

11. Messroghli DR, Radjenovic A, Kozerke S, Higgins DM, Sivananthan MU, Ridgway JP. Modified Look-Locker inversion recovery (MOLLI) for highresolution T1 mapping of the heart. Magn Reson Med. 2004;52(1):141-6.

12. Flett AS, Hasleton J, Cook C, Hausenloy D, Quarta G, Ariti C, et al. Evaluation of techniques for the quantification of myocardial scar of differing etiology using cardiac magnetic resonance. Jacc. 2011;4(2):150-6.

13. Dietrich O, Raya JG, Reeder SB, Reiser MF, Schoenberg SO. Measurement of signal-to-noise ratios in MR images: influence of multichannel coils, parallel imaging, and reconstruction filters. J Magn Reson Imaging. 2007;26(2):375-85.

14. Messroghli DR, Rudolph A, Abdel-Aty H, Wassmuth R, Kuhne T, Dietz R, et al. An open-source software tool for the generation of relaxation time maps in magnetic resonance imaging. BMC Med Imaging. 2010;10:16.

15. Messroghli DR, Walters K, Plein S, Sparrow P, Friedrich MG, Ridgway JP, et al. Myocardial T1 mapping: application to patients with acute and chronic myocardial infarction. Magn Reson Med. 2007;58(1):34-40.

16. Durmus T, Schilling R, Doeblin P, Huppertz A, Hamm B, Taupitz M, et al. Gadobutrol for magnetic resonance imaging of chronic myocardial infarction: intraindividual comparison with gadopentetate dimeglumine. Invest Radiol. 2012;47(3):183-8.

17. De Cobelli F, Esposito A, Perseghin G, Sallemi C, Belloni E, Ravelli S, et al. Intraindividual comparison of gadobutrol and gadopentetate dimeglumine for detection of myocardial late enhancement in cardiac MRI. AJR Am J Roentgenol. 2012;198(4):809-16.

18. Rudolph A, Abdel-Aty H, Bohl S, Boye P, Zagrosek A, Dietz R, et al. Noninvasive detection of fibrosis applying contrast-enhanced cardiac magnetic resonance in different forms of left ventricular hypertrophy relation to remodeling. J Am Coll Cardiol. 2009;53(3):284-91.

19. Assomull RG, Prasad SK, Lyne J, Smith G, Burman ED, Khan M, et al. Cardiovascular magnetic resonance, fibrosis, and prognosis in dilated cardiomyopathy. J Am Coll Cardiol. 2006;48(10):1977-85. 
20. Schulz-Menger J, Bluemke DA, Bremerich J, Flamm SD, Fogel MA, Friedrich $M G$, et al. Standardized image interpretation and post processing in cardiovascular magnetic resonance: Society for Cardiovascular Magnetic Resonance (SCMR) board of trustees task force on standardized post processing. J Cardiovasc Magn Reson. 2013;15:35

21. Knowles BR, Batchelor PG, Parish V, Ginks M, Plein S, Razavi R, et al. Pharmacokinetic modeling of delayed gadolinium enhancement in the myocardium. Magn Reson Med. 2008;60(6):1524-30.

22. Heiberg E, Sjogren J, Ugander M, Carlsson M, Engblom H, Arheden H. Design and validation of Segment-freely available software for cardiovascular image analysis. BMC Med Imaging. 2010;10:1.

23. Ugander M, Oki AJ, Hsu LY, Kellman P, Greiser A, Aletras AH, et al. Extracellular volume imaging by magnetic resonance imaging provides insights into overt and sub-clinical myocardial pathology. Eur Heart J. 2012; 33(10):1268-78.

24. Mongeon FP, Jerosch-Herold M, Coelho-Filho OR, Blankstein R, Falk RH, Kwong RY. Quantification of extracellular matrix expansion by CMR in infiltrative heart disease. JACC Cardiovasc Imaging. 2012;5(9):897-907.

25. Tao Q, Milles J, Zeppenfeld K, Lamb HJ, Bax JJ, Reiber JH, et al. Automated segmentation of myocardial scar in late enhancement MRI using combined intensity and spatial information. Magn Reson Med. 2010;64(2):586-94.

26. Bauner KU, Reiser MF, Huber AM. Low dose gadobenate dimeglumine for imaging of chronic myocardial infarction in comparison with standard dose gadopentetate dimeglumine. Invest Radiol. 2009:44(2):95-104.

27. Tumkosit M, Puntawangkoon C, Morgan TM, Clark HP, Hamilton CA, Ntim WO, et al. Left ventricular infarct size assessed with $0.1 \mathrm{mmol} / \mathrm{kg}$ of gadobenate dimeglumine correlates with that assessed with $0.2 \mathrm{mmol} / \mathrm{kg}$ of gadopentetate dimeglumine. J Comput Assist Tomogr. 2009:33(3):328-33.

28. Balci NC, Inan N, Anik Y, Erturk MS, Ural D, Demirci A. Low-dose gadobenate dimeglumine versus standard-dose gadopentate dimeglumine for delayed contrast-enhanced cardiac magnetic resonance imaging. Acad Radiol. 2006; 13(7):833-9

29. Schlosser T, Hunold P, Herborn CU, Lehmkuhl H, Lind A, Massing S, et al. Myocardial infarct: depiction with contrast-enhanced MR imaging-comparison of gadopentetate and gadobenate. Radiology. 2005;236(3):1041-6.

30. Bandettini WP, Kellman P, Mancini C, Booker OJ, Vasu S, Leung SW, et al. MultiContrast Delayed Enhancement (MCODE) improves detection of subendocardial myocardial infarction by late gadolinium enhancement cardiovascular magnetic resonance: a clinical validation study. J Cardiovasc Magn Reson. 2012;14(1):83.

31. Klem I, Weinsaft JW, Bahnson TD, Hegland D, Kim HW, Hayes B, et al Assessment of myocardial scarring improves risk stratification in patients evaluated for cardiac defibrillator implantation. J Am Coll Cardiol. 2012;60(5): 408-20.

32. Fluechter S, Kuschyk J, Wolpert C, Doesch C, Veltmann C, Haghi D, et al. Extent of late gadolinium enhancement detected by cardiovascular magnetic resonance correlates with the inducibility of ventricular tachyarrhythmia in hypertrophic cardiomyopathy. J Cardiovasc Magn Reson. 2010;12:30.

\section{Submit your next manuscript to BioMed Central and take full advantage of:}

- Convenient online submission

- Thorough peer review

- No space constraints or color figure charges

- Immediate publication on acceptance

- Inclusion in PubMed, CAS, Scopus and Google Scholar

- Research which is freely available for redistribution

Submit your manuscript at www.biomedcentral.com/submit 